\title{
Awareness and documentation of the teratogenic effects of valproate among women of child-bearing potential
}

\author{
Deirdre Mulryan, ${ }^{1}$ Anna Mclntyre, ${ }^{2}$ Colm McDonald, ${ }^{1,3}$ Sabina Feeney, ${ }^{2}$ Brian Hallahan ${ }^{1,3}$
}

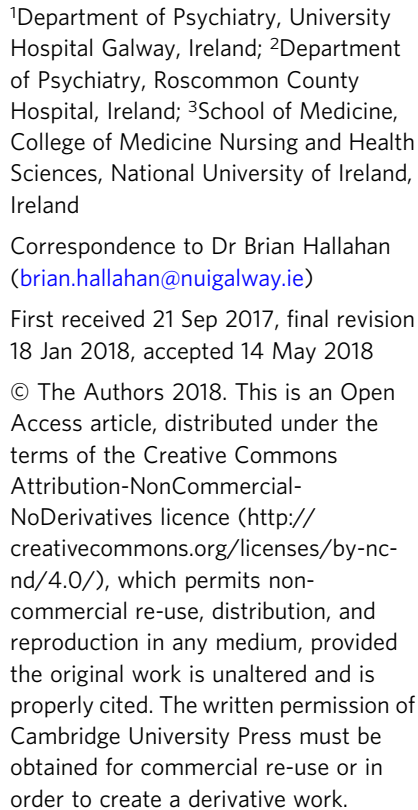

Aims and method We aimed to evaluate clinical note documentation of valproate prescribing and establish the level of knowledge among women of child-bearing potential regarding valproate-associated adverse effects, including teratogenesis, in a regional Irish mental health service.

Results Of the 42 women prescribed sodium valproate, $21.4 \%(n=9)$ had some documentation in relation to associated risks and 33.3\% $(n=14)$ described an awareness of these risks from consultation with their treating mental health team. On clinical interview, 9.5\% $(n=4)$ of individuals with clear documentation of the risks of teratogenesis described no such awareness. Augmentation with lithium was associated with greater awareness of the teratogenic risks of valproate $(P=0.011)$.

Clinical implications A clear description of the teratogenic risks of valproate and potential management strategies, including advice regarding contraception and supplementation with folic acid, should be clearly documented and provided repeatedly and in context to all women of child-bearing age who are prescribed valproate.

Declaration of interest None.

Keywords Valproate; folate; teratogenesis.
Valproate is a broad-spectrum anticonvulsant with wellestablished efficacy for the management of partial and generalised seizures, ${ }^{1}$ acute (hypo)mania and prophylaxis of (hypo)manic episodes both as a monotherapy or in combination with other psychotropic agents. ${ }^{2}$ In addition, valproate can be utilised for the prophylaxis of depressive episodes in bipolar disorder, ${ }^{2}$ although this reported therapeutic effect is of lesser magnitude. ${ }^{3}$

\section{Risk of teratogenesis}

However, valproate use in women of child-bearing potential is associated with a wide range of neurodevelopmental deficits, ${ }^{4}$ with an approximate risk of teratogenesis of $7 \%^{5}$ and $70 \%$ of teratogenesis malformations consisting of neural tube defects (NTDs), ${ }^{6}$ which is a 20 -fold increase compared with the general population. ${ }^{7}$ NTDs occur when the embryonic neural tube fails to completely close during development, and include spina bifida and anencephaly. ${ }^{8}$ In addition to NTDs, valproate use in pregnancy is also associated with an increased incidence of cleft lip and palate, cardiovascular abnormalities, genitourinary defects, endocrine disorders, cognitive impairment, developmental delay and autism spectrum disorders in the foetus. ' A 'foetal valproate syndrome' has also been described and includes a constellation of physical traits consistently observed in foetuses exposed in utero to valproate such as a flat nasal bridge, epicanthal folds, small upper lids and a downward-turned mouth. ${ }^{10,11}$ Potential contributory factors to the teratogenicity of valproate have been reported and include the number of co-administered medications, higher dosages of valproate, differences in maternal and/or foetal metabolism, gestational age of the foetus at exposure to valproate and hereditary susceptibility. ${ }^{12,13}$

The teratogenic risk associated with valproate has resulted in significant caution with its use, with the summary of product characteristics for sodium valproate and the related compound semi-sodium valproate and the National Institute of Health and Care Excellence (NICE) guidelines of 2014 stating that valproate should not be initiated in women of child-bearing potential without specialist advice or unless other treatments are not tolerated or are ineffective (https: \\www.gov.uk/government/publications/toolkit-on-the-risks-of-valproate-medicines-in-femalepatients) 


\section{Awareness of teratogenic risk}

Given the risks associated with valproate use during pregnancy, awareness of these risks and a consideration of what precautions are required in relation to the use of valproate in women of child-bearing potential is important. However, previous research has demonstrated low rates of documentation in clinical notes of advice from mental health staff regarding both the teratogenic risks of valproate and the importance of both contraception and folic acid use. $^{14-17}$

\section{Aims}

Consequently, in this study we wanted to establish if women of child-bearing potential attending a mental health service in Ireland were prescribed valproate and ascertain the indications for this treatment. In addition, we wanted to assess the rate of documentation of advice relating to the teratogenic risks of valproate and importance of the use of contraception and folic acid. We wanted to establish the awareness of women of child-bearing potential who are prescribed valproate regarding the teratogenic risks of this agent, and the potential benefits of contraception and folic acid. We also wanted to enquire establish how those with such awareness attained this information.

\section{Method \\ Participants}

This study was undertaken in an Irish mental health service region with an approximate population of 250000 people. All female adult patients attending this mental health service in January 2016 were screened $(n=1620)$ to ascertain if they were being treated with valproate. Community mental health team staff members, including consultant psychiatrists, non-consultant psychiatry hospital doctors, community mental health nurses, allied health professionals and administrators, were contacted to ascertain potential participants. Inclusion criteria included women aged 1849 years who had been in receipt of care from the mental health service for $>1$ year and had been treated with valproate.

Exclusion criteria included individuals $<18$ years of age, the presence of an intellectual disability (IQ<70), a diagnosis of dementia and the presence of acute psychosis. None of the 42 individuals identified as receiving treatment with valproate fulfilled these exclusion criteria. Clinical diagnoses were attained utilising ICD-10 diagnostic criteria after consultation with the relevant treating consultant psychiatrist and a full clinical chart review. ${ }^{18}$

Ethical approval was attained from the Galway University Hospitals Research Ethics Committee (C.A. 841) and the study was conducted in accordance with the Helsinki declaration on research ethics. Written informed consent was attained from each participant for clinical note review, clinician contact and semi-structured interview. On completion of the semi-structured interview, psychotherapeutic support was offered to each participant.

\section{Chart review}

A retrospective chart review was undertaken of the lifetime clinical notes of included individuals. Clinical notes were examined in depth (by D.M., A.M. and S.F.) and included all handwritten, typed and electronically generated entries pertaining to in-patient admissions to one of three acute psychiatric in-patient units; attendance at the different day hospitals, day centres and out-patient clinics in the region; correspondence to and from general practitioners and specialists (including neurologists) and all prescription records.

Demographic data collected included gender, age and socioeconomic, employment, marital and domiciliary status. Clinical data included diagnosis, prescription of valproate, time duration and dose of valproate prescribed and prescription of any concomitant psychotropic medication (mood stabilisers, anticonvulsants, antidepressants, antipsychotics, anxiolytics, hypnotics and anticholinergic agents). We also ascertained if children were conceived to patients when prescribed valproate, and if any foetal abnormalities were recorded. Data pertaining to the documentation of the risk of teratogenicity or congenital malformations secondary to valproate, and precautions including adequate contraception and use of folic acid were recorded.

\section{Semi-structured interview}

All 42 identified participants participated in a semistructured interview. Information gathered included awareness of the teratogenic risk of valproate; how, when and by whom participants were first informed of this risk (if informed); advice regarding use of contraception; wishes regarding pregnancy before or during treatment with valproate; undertaking a pregnancy test before commencement of valproate and advice regarding, or prescription of folic acid. We also enquired if participants became pregnant or conceived when treated with valproate and ascertained what advice regarding treatment with valproate was attained from their treating clinician during their pregnancy (see Appendix 1, Semi-Structured Interview Topic Guidelines).

\section{Data analyses}

Statistical analyses were performed with SPSS 22.0 for Windows (SPSS Inc., New York, USA). We utilised the Student's $t$-test for parametric data and the $\chi^{2}$-test for nonparametric categorical data, where appropriate. Linear regression was conducted to ascertain the effect of age, dose of valproate and duration of treatment in relation to clinical documentation or awareness of the teratogenic risks of valproate.

\section{Results}

\section{Demographic and clinical data}

Demographic and clinical data are described in Table 1. Of note, the most common diagnosis was bipolar disorder $(33.3 \%, n=14)$ and the most common comorbid psychotropic 


\begin{tabular}{|c|c|}
\hline Variable & $n(\%)$ or mean (s.d.) [range] \\
\hline \multicolumn{2}{|l|}{ Employment status } \\
\hline In third-level education ${ }^{a}$ & $3(7.1)$ \\
\hline Employed & $10(23.8)$ \\
\hline Unemployed & $26(69.0)$ \\
\hline \multicolumn{2}{|l|}{ Relationship status } \\
\hline Single & $27(64.3)$ \\
\hline In a relationship & $4(9.5)$ \\
\hline Married & $10(23.8)$ \\
\hline Divorced/separated & $1(2.4)$ \\
\hline \multicolumn{2}{|l|}{ Living arrangement } \\
\hline Alone & $2(4.8)$ \\
\hline With parents or siblings & $12(28.6)$ \\
\hline With partner or spouse & $14(33.3)$ \\
\hline In supported accommodation & $14(33.3)$ \\
\hline \multicolumn{2}{|l|}{ Socioeconomic class $^{\mathrm{b}}$} \\
\hline | or II & $10(23.8)$ \\
\hline III & $0(0.0)$ \\
\hline IV & $30(71.4)$ \\
\hline $\mathrm{V}$ & $2(4.8)$ \\
\hline \multicolumn{2}{|l|}{ Mental disorder (ICD-10) } \\
\hline Bipolar disorder & $14(33.3)$ \\
\hline Recurrent depressive disorder & $12(28.6)$ \\
\hline Schizophrenia $^{c}$ & $7(16.7)$ \\
\hline Schizoaffective disorder & $6(14.3)$ \\
\hline $\begin{array}{l}\text { Emotionally unstable personality } \\
\text { disorder }\end{array}$ & $3(7.1)$ \\
\hline \multicolumn{2}{|l|}{ Other psychotropic agents } \\
\hline Antipsychotics & $30(71.4)$ \\
\hline Antidepressants & $20(47.6)$ \\
\hline Lithium & $6(14.3)$ \\
\hline Anti-epileptics & $5(11.9)$ \\
\hline Benzodiazepines & $8(19.0)$ \\
\hline Hypnotics & $5(11.9)$ \\
\hline Anticholinergics & $3(7.1)$ \\
\hline Age at interview & $41.5(6.7)[24-49]$ \\
\hline Sodium valproate dose, $\mathrm{mg} /$ day & $923(434)[300-2000]$ \\
\hline Duration of treatment, years & 7.59 (3.92) [1-14] \\
\hline
\end{tabular}

a. In this study, undertaking a university course

b. Socioeconomic class was scored based on NRS grading: I = upper-middle class/higher managerial, administrative or professional; $\|=$ intermediate managerial, administrative or professional; $I I I=$ supervisory or junior managerial or skilled manual workers; IV = semi-skilled or unskilled manual workers, $V=$ unemployed, on social welfare, minimum-wage workers. c. Used as a prophylactic treatment for seizure control due to treatment of schizophrenia with clozapine.

medications prescribed were antipsychotic agents (71\%). In addition, $16.7 \%$ of individuals $(n=7)$ were treated with valproate for prophylaxis of epilepsy due to treatment with high dosages of clozapine.

\section{Clinical documentation}

Documentation of risks associated with valproate in pregnancy were present in $21.4 \%(n=9)$ of the clinical files and included advice on contraception $(16.7 \%, n=7)$, the specific teratogenic risks of valproate $(9.5 \%, n=4)$ and use of folic acid $(2.4 \%, n=1)$. No individuals had a documented pregnancy test before initiation of valproate.

A range of sociodemographic factors, including maternal age at interview $(B=-0.101, P=0.54)$, or clinical variables, including dose of valproate $(B=0.205, P=0.21)$ and treatment duration $(B=0.127, P=0.45)$, were not associated with clinical documentation of the teratogenic risks of valproate. All nine individuals with a comorbid diagnosis of epilepsy had no clinical documentation of the risks of valproate, although this was not statistically significant when compared with those treated with valproate for a mental health disorder ( $P=0.17$, Fisher's exact test). Valproate augmentation with psychotropic medication was present in $95.2 \%(n=40)$ of individuals. Three of the six individuals treated with lithium had written documentation of the risks of valproate.

\section{Structured interview}

On interview, $33.3 \%(n=14)$ of participants demonstrated some awareness of the risk of valproate, with $19.0 \%(n=8)$ aware of specific teratogenic risks, requirement for contraceptive use and need for a pregnancy test before valproate initiation, and $16.7 \%(n=8)$ aware of the need for folic acid use when taking valproate. All but one of these participants stated that they had acquired this information from their treating consultant psychiatrist. Nine (64.3\%) individuals who stated that they were informed of the risks of valproate as acquired from their treating clinician had no documented information detailing this advice/information in their clinical files. Overall, 18 (42.9\%) individuals had either documented data in their clinical notes or stated that their mental health team had advised them on at least some of the risks of valproate for women of child-bearing potential. Four individuals (9.5\%) had documented advice in their clinical notes but had no awareness of same (on clinical interview).

Regression analysis showed that younger age $(B=-0.315$, $P=0.042$ ) was associated with a greater awareness of the teratogenic risks of valproate. Additionally, individuals augmented with lithium were more likely to be aware of the teratogenic risks of valproate $(83 v .25 \%, P=0.011)$.

\section{Pregnancies on valproate}

Four participants had pregnancies (all uncomplicated) when treated with valproate. Three continued on the same treatment and dosage, with documentation that they should continue because of the risk of relapse of their seizure disorder. There was no documentation in these clinical files in relation to the risks of valproate in pregnancy or advice regarding contraception or folic acid use. The fourth participant had a diagnosis of bipolar disorder and had documentation to discontinue valproate (at 4 weeks' gestation), but there was no documented advice to commence folic acid. On structured interviews, in addition to the individual who 
discontinued valproate treatment, two of the other three participants stated that their consultant psychiatrist advised them to commence folic acid, but there was no documentation in the clinical files in relation to this.

\section{Discussion}

This is the first study to date, to our knowledge, to review both clinical documentation and undertake semi-structured interviews with women of child-bearing potential who were prescribed valproate. Either clear documentation of the risks of valproate or an awareness of these risks after discussion with their treating clinical team was present for $43 \%$ of individuals.

Our findings of poor clinical documentation of specific risks of valproate are consistent with previous research, with rates of $16-29 \%$ noted for documentation of risks of teratogenesis or the provision of advice in relation to contraception for women of child-bearing potential who are prescribed valproate. ${ }^{14,16}$ Similarly, documented advice in clinical notes relating to the use of folic acid has previously also been noted to be particularly low (4\%). ${ }^{16}$ Our findings in relation to patient awareness of teratogenic risks of valproate are also consistent with previous studies (17-28\%); ${ }^{12,17}$ however, awareness of the need for contraceptive use was lower in our patient cohort compared with a number of previous studies $(55-67 \%){ }^{15,17}$

It is probable that the majority of patients examined in this study were provided with verbal advice regarding the risks of valproate. Two-thirds of patients who said they had been advised of the risks of valproate had no clinical documentation relating to this advice. Some participants, despite clinical documentation stating they had been informed of the risks of valproate, stated at the time of interview that they had no awareness of such a discussion (which may potentially be related to recall bias). This highlights the need for information (including in a written format) relating to the teratogenic risks of valproate to be provided repeatedly and in context to patients to ensure awareness of these risks. This may be particularly important for this cohort of patients who are being treated for bipolar disorder or schizoaffective disorder, where impulsive behaviour including risky sexual behaviour may occur during a (hypo)manic episode. ${ }^{14}$ The lack of documentation in mental health clinical notes of advice to individuals treated for epilepsy with valproate may be secondary to the fact that mental health staff had not initiated this treatment, with evidence suggesting that clinicians are less likely to inform patients of medications adverse events if they have not initiated the particular medication themselves. ${ }^{17}$ However, three individuals diagnosed with epilepsy described being advised of the risks of valproate by their mental health team, and it is probable that some participants additionally attained advice from other clinicians (i.e. neurologists, general practitioner) relating to the teratogenic risks of valproate. The provision of written information, and advice on appropriate resources that gives accurate advice in relation to the risks and benefits of valproate and precautions required with its use is suggested. Information recently produced by the National Clinical Programme for Medicine Management is one potential option in this regard. ${ }^{19}$

This study highlights the low rates of documentation of contraceptive use or advice regarding folic acid. Consultations with mental health staff (preconception consultations) in relation to the risks and benefits of continuation or discontinuation of medications, including for women diagnosed with bipolar disorder, have previously been demonstrated to influence both choices regarding conception and treatment. ${ }^{20}$ This study suggests that such consultations, focusing on issues of conception and pharmacotherapeutic treatment, should be considered for all women of child-bearing potential (even if not in a significant relationship) who are prescribed valproate. NICE guidelines ${ }^{21}$ state that valproate should not be offered to women of child-bearing potential for either the management of acute mood episodes or the long-term management of bipolar disorder, and if already prescribed, should gradually be discontinued because of the teratogenic risks of valproate. Additionally, folic acid should always be prescribed to women of child-bearing potential who are prescribed valproate, ${ }^{22}$ with levels to be undertaken both before commencement of valproate and on a 6-monthly basis.

\section{Limitations}

This study includes a number of limitations, including that females of child-bearing age who were under 18 years old were not included. Findings were attained from one region in Ireland and may not be representative of other regions or other jurisdictions; however, many of the findings are consistent with those noted in other jurisdictions and thus may indeed be generalisable. Clinical notes from other practitioners, including neurologists and general practitioners (excluding correspondence from them as detailed in the mental health clinical notes), were not examined or available. Finally, the semi-structured interviews conducted were retrospective in nature, and thus potentially liable to recall bias. Recall bias may partly explain the discrepancy we found, where documentation detailing the teratogenic risk of valproate was noted but not recollected by participants. It is probable that other non-mental health service clinicians may have disseminated information relating to the risks of valproate; however, this was not evident on clinical interview and this study principally investigated documentation in mental health clinical records.

\section{Appendix 1}

\section{Semi-Structured Interview Topic Guidelines}

1. Are you aware of any risks associated with the use of sodium valproate?

Please list these risks

Please identify how you became aware of these risks 
2. Are you specifically aware of any problems this medication has in relation to pregnancy and foetal development?

What have you been told?

Who told you?

When were you told?

3. Was your wish or likelihood of becoming pregnant ascertained before commencing therapy?

4. Was contraceptive advice offered?

5 . Was use of contraception confirmed before commencing treatment?

6. Was a pregnancy test done before commencing sodium valproate?

7. Were you advised regarding the use of folic acid supplementation?

\section{About the authors}

Deirdre Mulryan Senior Registrar, University Hospital Galway, Galway Roscommon Mental Health Services, Ireland; Anna McIntyre, Registrar in Psychiatry, University Hospital Galway, Ireland; Colm McDonald, Professor of Psychiatry, School of Medicine, College of Medicine Nursing and Health Sciences, National University of Ireland Galway, Ireland and Consultant Psychiatrist, University Hospital Galway, Galway Roscommon Menta Health Services, Ireland; Sabina Feeney, Consultant Psychiatrist, Roscommon County Hospital, Ireland; Brian Hallahan, Senior Lecturer, School of Medicine, College of Medicine Nursing and Health Sciences, National University of Ireland Galway, Ireland and Consultant Psychiatrist, University Hospital Galway, Galway Roscommon Mental Health Services, Ireland

\section{References}

1 Loescher W, Levy RH, Mattson RH, Meldrum BS, Perucca E, (eds). Valproic acid mechanisms of action. In Antiepileptic Drugs: 767-79. Lippincott Williams \& Wilkins, 2002

2 Fountoulakis KN, Vieta E, Sanchea-Morena J, Kaprinis SG, Goikolea JM Kaprinis GS. Treatment guidelines for bipolar disorder: a critical review. J Affect Disord 2005; 86: 1-10.

3 Bowden CL. Valproate. Bipolar Disord 2003; 5: 189-202.

4 Meador KJ. Neurodevelopmental effects of antiepileptic drugs. Current Neurol Neurosci Rep 2002; 2: 373-8.

5 Tomson T, Marson A, Boon P, Canevini MP, Covanis A, Gaily E, et al. Valproate in the treatment of epilepsy in girls and women of childbearing potential. Epilepsua 2015; 56(7): 1006-19.

6 Omtzigt J, Los FJ, Hagenaars AM, Stewart PA, Sachs ES, Lindhout D. Prenatal diagnosis of spina bifida aperta after first trimester valproate exposure. Prenat Diagn 1992; 12: 893-7.

7 Hrubec TC, Yan M, Keying Y, Salafia CM, Holladay SD. Valproic acid induced fetal malformations are reduced by maternal immune stimulation with granulocyte-macrophage colony-stimulating factor or interferon Y. Anat Rec Discov Mol Cell Evol Biol 2006; 288: 1303-9.

8 Cragan JD, Roberts HE, Edmonds LD, Khoury MJ, Kirby RS, Shaw GM et al. Surveillance for anencephaly and spina bifida and the impact of prenatal diagnosis-United States, 1985-1994. MMWR CDC Surveill Summ 1995; 44: 1-13.

9 Adab N, Kini U, Vinten J, Ayres J, Baker G, Clayton-Smith J, et al. The longer-term outcome of children born to mothers with epilepsy. J Neurol Neurosurg Psychiatry 2004; 75: 1575-83.

10 Chiu CT, Wang Z, Hunsberger JG, Chuang DM. Therapeutic potential of mood stabilizers lithium and valproic acid: beyond bipolar disorder. Pharmacol Rev 2013; 65: 105-42.

11 DiLiberti JH, Farndon PA, Dennis NR, Curry CJ. The fetal valproate syndrome. Am J Med Genet 1984; 19: 473-81.

12 Meador KJ, Baker GA, Finnell RH, Kalayjian LA, Liporace JD, Loring DW et al. In utero antiepileptic drug exposure: fetal death and malformations. Neurology 2006; 67: 407-12.

13 Alsdorf R, Wyszynski DF. Teratogenicity of sodium valproate. Expert Opinion Drug Saf 2005; 4: 345-53.

14 James L, Barnes TRE, Lelliott P, Taylor D, Paton C. Informing patients of the teratogenic potential of mood stabilising drugs: a case note review of the practice of psychiatrists. J Psychopharmacol 2007; 21 815-9.

15 Langan J, Perry A, Oto M. Teratogenic risk and contraceptive counselling in psychiatric practice: analysis of anticonvulsant therapy. BMC Psychiatry 2013; 13: 234

16 Attura $\mathrm{H}$, Odelola A. Valproate prescribing in women of child bearing age: an audit of clinical practice. Adv Psychiatry 2015; 2015 520784.

17 James L, Paton C, Lelliott P, Barnes TRE, Taylor D. Mood stabilizers and teratogenicity-prescribing practice and awareness among practicing psychiatrists. J Ment Health 2009; 18: 137-43.

18 ICD-10 Classification of Mental and Behavioural Disorders, Churchill Livingstone, Elsevier Limited, 1994.

19 National Clinical Programme for Epilepsy, Mental Health and Medicines Management in consultation with Health Promotion and Regulation Authority. Use of Valproate Toolkit. Health Service Initiative, 2016 (https://www.hse.ie/eng/about/who/cspd/ncps/medicines-management/patient-information/valproate)

20 Viguera AC, Cohen LS, Bouffard S, Whitfield TH, Baldessarini RJ. Reproductive decisions by women with bipolar disorder after pregnancy psychiatric consultation. Am J Psychiatry 2002; 159: 2102-4.

21 National Institute for Health and Care Excellence. Bipolar Disorder: Assessment and Management, Clinical Guidance 185. NICE, 2014 (https://www.nice.org.uk/guidance/cg185/chapter/Update-information.org.uk).

22 National Institute for Health and Care Excellence. Epilepsies: diagnosis and management Clinical Guidance 137. NICE, 2012 (https://www. nice.org.uk/guidance/cg137/chapter/1-Guidance\#women-and-girlswith-epilepsy).

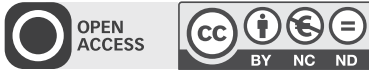

